\title{
NOVEL ASPECTS TO THE STRUCTURE OF RABBIT ARTICULAR CARTILAGE
}

\author{
I. ap Gwynn ${ }^{1 *}$, S. Wade 1 , K. Ito ${ }^{2}$ and R.G. Richards ${ }^{2}$ \\ ${ }^{1}$ Institute of Biological Sciences, The University of Wales, Aberystwyth, Wales SY23 3DA \\ ${ }^{2} \mathrm{AO}$ Research Institute, Clavadelerstrasse, CH-7270 Davos, Switzerland.
}

\begin{abstract}
Applying cryo and modified chemical preparation techniques, mainly for scanning electron microscopy, revealed entirely new aspects to the structure of the radial zone of rabbit tibial plateau articular cartilage. The aggrecan component of the extracellular matrix was contained radially in columns, each with a diameter of $1-3 \mu \mathrm{m}$, by a tightly packed matrix of collagen fibrils. The collagen fibrils were arranged radially, some straight and others in an opposed spiral arrangement, with regularly repeating patterns. This organization existed in the regions surrounding the columns of chondrocytes, known as chondrons. The load bearing property of the tissue was explained by the directed flow and containment of the interstitial fluid, modulated by the protein-carbohydrate complexes, along these collagen bounded tubular structures. The reason why such a structure has not been described previously may be that it is not retained by aldehyde fixation followed by dehydration, the method commonly used for tissue preparation for electron microscopy.
\end{abstract}

Key Words: Ultrastructure, rabbit, articular cartilage, collagen, proteoglycans.

\footnotetext{
*Address for correspondence:

Iolo ap Gwynn,

Institute of Biological Sciences,

The University of Wales,

Aberystwyth,

Wales SY23 3DA.
}

Telephone number: $+44(0) 1970622324$

FAX number: $+44(0) 1970622350$

E-mail: iag@aber.ac.uk

\section{Introduction}

There are substantial gaps in our knowledge of articular cartilage (AC) structure. Traditional preservation techniques result in what are believed to be acceptable microscopic images. The application of alternative protocols revealed a different picture, which may contribute to a better understanding of the functional properties of the tissue.

Tibial AC is divided vertically into the calcified, radial and superficial zones (Figure 1) (Clarke, 1971; Lane and Weiss, 1975; Buckwalter et al., 1987; Ratcliffe and Mow, 1996). The dry weight of the tissue contains about two thirds collagen, being mostly type II but with some III, V, VI, IX, X and XI (Eyre et al., 1987; Eyre, 2001; Young et al., 1995; Morrison et al., 1996; Young et al.,

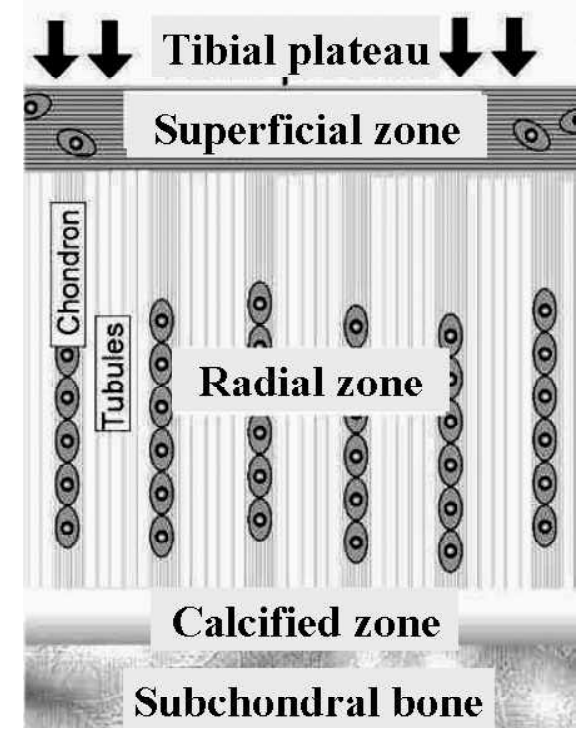

Figure 1: Diagrammatic representation of zones that were identifiable in articular cartilage. The superficial zone, next to the tibial plateau, had spongy collagen architecture with a degree of fibril orientation in the direction parallel to the articulating surface. Chondrocytes appeared scattered throughout this zone. In the adult rabbit tibial plateaux it was 30-40 $\mu \mathrm{m}$ thick. Immediately overlying the bone was the calcified zone. Between the calcified and superficial zones collagen fibrils were generally oriented in the radial direction, hence the description radial zone. In this zone there were columns of chondrocytes, in between which were regions in which collagen fibrils were very tightly packed together surrounding arrays of tubules. 
2000). The precise roles of the different types have yet to be understood. Within the collagen matrix, there are several types of chondrocytes. Those in the superficial zone are distributed through the region. The cells of the radial zone are different, being arranged in vertical columns, called chondrons (Poole et al., 1987). Benninghoff(1925) first described the 'gothic arch or arcade' type morphology in AC. This model is still generally accepted (Nötzli and Clark, 1997; Kääb et al., 1998). Scanning electron microscopy (SEM) of chemically fixed tissue, suggests that the collagen fibrils associate to form a columnar arrangement of radial zone fibres, perpendicular to the surface, that arch over and enter the superficial zone oriented parallel to the surface (Clark, 1991; Williams et al., 1999). There are significant structural differences between adult and immature AC (Bayliss et al., 2000). The precise timing of the maturation process may also differ considerably from species to species. We used the term 'collagen fibril' to describe the smallest structural element commonly observed by electron microscopy (diameter approx. 30nm). Accumulations of such fibrils could then be described as a 'fibre'. The larger units of structure, comprising the whole collagen network surrounding the chondrons (on a scale of $10 \mu \mathrm{m}$ or more, in diameter), we referred to as columns.

Proteoglycans (PG), composing about $30 \%$ of tissue dry weight, are entangled with each other and other molecules to form a large complex network (Poole et al., 1982). This network could be bound to the collagen network, or contained and entangled within it. The PGs are strongly hydrophilic, form gels at very low concentrations in water, carry many negative charges and consequently attract cations. Any attracted cations are osmotically active and consequently attract more water, which makes up $60-80 \%$ of the wet weight of the living tissue. The osmotic pressure generated by PGs in healthy tissue is at least 2atm (Urban et al., 1979; Basser et al., 1998) that would present serious problems when attempting to apply conventional chemical fixation techniques to the tissue. Collagen fibrils have a very high tensile strength, but with little ability to withstand compression. It is the hydrated PG that has this capability, provided it is immobilised and contained in a collagen matrix. With loading, the applied pressure often exceeds the osmotic swelling pressure, driving fluid out of the matrix. However, the low permeability of PGs resists the flow of fluid relative to the immobilised PGs, providing a highly damped system.

Transmission electron microscopy (TEM) of fixed and embedded as well as high-pressure frozen sections of bovine tissue reveals detailed ultrastructure of the collagen and PG (Hunziker et al., 1996). Individual collagen fibrils are bound together in some areas. Due partially to the section thickness used $(50-100 \mathrm{~nm})$, relative to the micron-level of collagen fibril organisation, TEM images fail to reveal the three-dimensional structure of the tissue. Applying conventional chemical fixation protocols, used for TEM specimen preparation, to a tissue with such highly unusual $\mathrm{pH}$ and osmotic properties, should result in considerable ultrastructural disruption. Hunziker et al. (1996) recognise this and show how cryo-techniques can be used to overcome this problem. The application of TEM to dissected tissue provides some indication of an organised coiled arrangement to the collagen fibrils in the radial zone (Broom, 1986; Chen and Broom, 1998).

SEM provides information on the gross morphological features of the tissue, including the effect of loading (Clark, 1991; Jeffery et al., 1991; Teshima et al., 1995; Richards and Kääb, 1996; Nötzli and Clark, 1997; Kääb et al., 1999a). The 'fibres' of the radial zone, adjacent to loaded regions are not deformed laterally to any significant extent. As there is no evidence for fluid transfer through the calcified region, most of the incompressible interstitial fluid would have to be displaced in the radial direction. None of the accepted structural models (Benninghof, 1925; Clark, 1991) explain satisfactorily this anisotropy in resistance to fluid movement and it is suggested that they need re-examination (Hunziker et al., 1997).

The much higher resolution of field emission scanning electron microscope (FESEM) study of the radial zone of freeze-substituted adult rabbit tissue reveals a level of collagen fibril organization that would provide a barrier to lateral fluid movement. In between the chondrons, the radial zone is seen as an array of tubules, whose walls are composed of a compact matrix of tightly packed collagen fibrils (ap Gwynn et al., 2000). The tubular structure appears to confine the chondrocytes to a vertical chondron column arrangement. The $30-40 \mu \mathrm{m}$ thick superficial zone has a more spongy appearance, with pore sizes of the order of 500nm diameter, with fibrils arranged in a direction parallel to the articulating surface. The application of modified chemical fixation protocols resulted in the preservation of a similar structural arrangement to that seen following the application of cryo-methods. The general arrangement of the radial zone components envisaged is represented in Figure 2. We provide further evidence to support this interpretation.

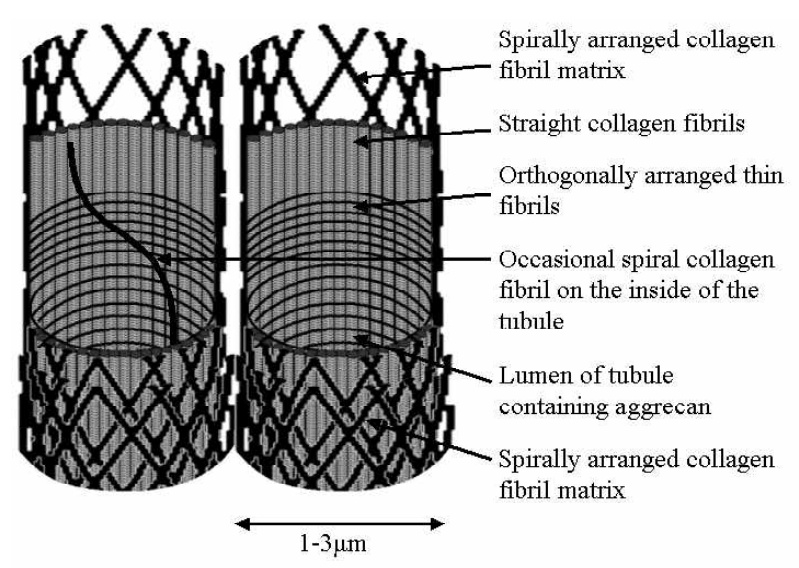

Figure 2: Diagrammatic representation of the suggested structural arrangement of the collagen and proteoglycans in the radial zone of rabbit tibial articular cartilage. 


\section{Materials and Methods}

Knee joints from adult White New Zealand and wild Welsh rabbits were obtained. No morphological differences were found between the AC from the two types of rabbit. Each joint was cut open with a scalpel to reveal the tibial plateau. The tibial plateau region was cut off the end of the tibia, using a carborundum disk cutter. A Meisinger (Düsseldorf, Germany) type 224 RF dental trephine burr, attached to a small electrical drill was used to extract $1 \mathrm{~mm}$ diameter cores, traversing the whole thickness of the tissue from tibial plateau to the sub-chondral bone. Cores were taken from plateau areas where the femoral condyle applied pressure. The specimen was then frozen rapidly in liquid propane at liquid nitrogen temperature, fractured under liquid nitrogen using a special jig (Figure 3), freezesubstituted in 1:2 acetone:methanol containing 10\% acrolein and $0.2 \%$ tannic acid - followed by anhydrous acetone, critical point dried in carbon dioxide using a Polaron E3000 apparatus (Agar Scientific, Stansted, UK), sputter coated with $10 \mathrm{~nm}$ platinum or $8 \mathrm{~nm}$ gold-palladium (4:1), using a Baltec (Balzers, Liechtenstein) Med 020 unit, and examined in an FESEM (Hitachi, Tokyo, Japan; s-4100 or s-4700) operated at an appropriate accelerating voltage (ap Gwynn et al., 2000). Other samples were treated, instead of applying critical point drying, by transferring through an ethanol series into Lowicryl K11M embedding resin (Agar Scientific). Infiltration of the resin was carried out over a period of three weeks. Following polymerisation of the resin ultrathin sections were cut and viewed using a JEOL (Tokyo, Japan) 100CX TEM, operated at $100 \mathrm{kV}$ accelerating voltage.

For microwave enhanced chemical fixation of tissue for TEM $1 \mathrm{~mm}$ cores were taken, as described above. The specimens were then placed in a solution of $2.5 \%$ glutaraldehyde in $0.1 \mathrm{M}$ phosphate buffer ( $\mathrm{pH} 7.2)$, and exposed to bursts of microwave energy (set at the minimum power setting $[10 \%]$, temperature sensor in the container next to the specimen, $300 \mathrm{ml}$ water buffer) until the fixative solution temperature reached $40^{\circ} \mathrm{C}$ (Richards and Kääb, 1996; Kääb et al., 1999b). The specimens were then further fixed for 30 minutes in the buffered glutaraldehyde solution, at $20^{\circ} \mathrm{C}$, before transferring to a $1 \%$ osmium tetroxide solution for 1 hour at $20^{\circ} \mathrm{C}$. Dehydration was subsequently carried out in ethanol series and the specimens were either embedded in TAAB (Aldermaston, Berks, UK) embedding resin (infiltration for 4 days) or critical point dried. Ultrathin sections of the embedded material were observed using a JEOL 100CX TEM, operated at $100 \mathrm{kV}$ accelerating voltage. The microwave stage was also omitted, and the initial glutaraldehydebased fixative was applied to the tissue for 2 hours at $20^{\circ} \mathrm{C}$, buffered at various $\mathrm{pH}$ values from 4.0 to 10.0 before dehydrating and critical point drying or resin embedding the specimens.

Fully dried specimens, following chemical or cryopreparation, were also cooled by immersion in liquid nitrogen on the fracturing jig and further fractured. To avoid damage to the structure by condensing water, on the newly fractured cold specimen, the samples were kept under liq-

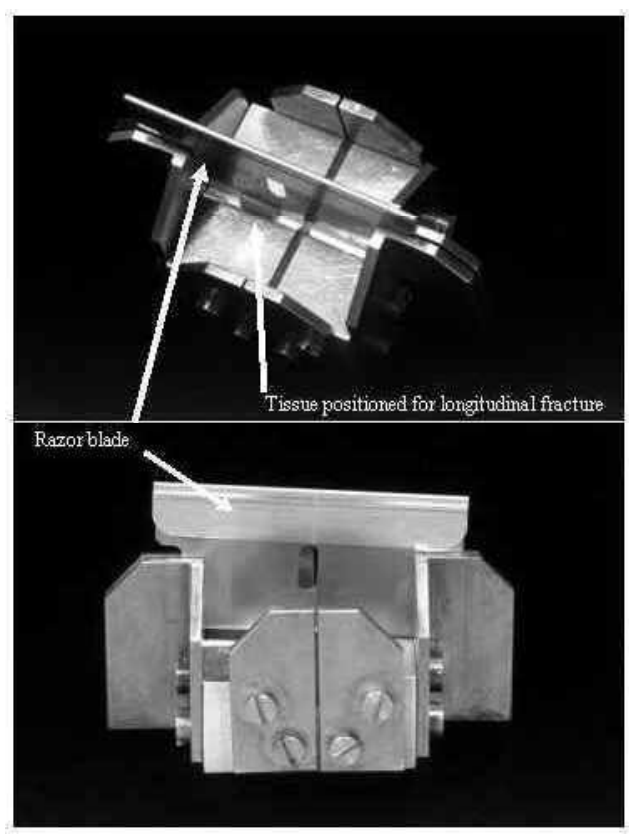

Figure 3: Fracturing jig device designed for controlling the direction of fractures in cores of articular cartilage (designed and built by P.C. Lloyd). Cores were placed in the groove of the jig, a razor blade was placed in the appropriate guides and the whole assembly cooled in liquid nitrogen. The blade was then struck sharply with a hammer.

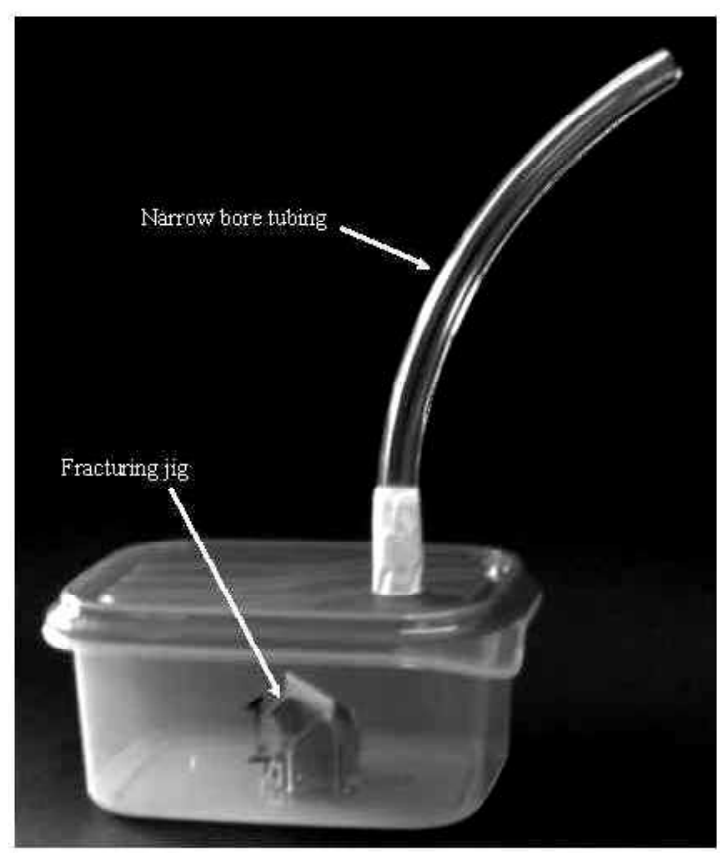

Figure 4: Warming-up device for holding the fracturing jig and fractured sample until the liquid nitrogen had all boiled off and the whole assembly reached room temperature. The working principle was that any moist air present in the closed container was driven out of the narrow bore tubing by the boiling liquid nitrogen. The specimen was therefore held in dry nitrogen until it had reached room temperature and there was no danger of atmospheric moisture condensing on a cold specimen, destroying the surface ultrastructure. 
uid nitrogen in a small plastic container. A tightly sealing lid, to which a $15 \mathrm{~cm}$ long piece of $5 \mathrm{~mm}$ bore plastic tubing had been attached, was then placed on the container (Figure 4). Evaporating nitrogen drove out all the air, through the narrow tube, as it boiled off. Once all the liquid nitrogen had boiled off, the chamber only contained gaseous nitrogen, and no moist air could return along the whole length of the thin tube to harm the specimen surface. Once the whole container, including the specimen, had warmed up to room temperature it could be opened and the specimen removed for mounting onto SEM stubs and sputter coated.

For confocal laser scanning microscopy, approximately 1-2 mm thick vertical sections were cut through the tissue, using a sharp razor blade. Images were taken, through a standard 1.5 thickness cover-slip, at an optical section thickness of $1 \mu \mathrm{m}$, with a $10 \mathrm{x}$ objective lens. The microscope used was a BioRad (Hemel Hempsted, UK) MRC1024, with a $100 \mathrm{~mW}$ argon laser generating illumination at $488 \mathrm{~nm}$ (the natural auto-fluorescence wavelength for collagen). The emission collection wavelength was $580 \mathrm{~nm} \pm 32 \mathrm{~nm}$.

\section{Results and Discussion}

Where the radial zone tubules were fractured longitudinally the luminal walls were seen to be composed of tightly packed longitudinal collagen fibrils that appeared to run the length of the tubule, each fibril being about $30 \mathrm{~nm}$ in diameter (Figures 5 to 7). The tight packing and orientation of the fibrils was consistent with the well-established birefringent properties of the collagen in the radial zone (Benninghof, 1925; Kääb et al., 1998). Only such tightly packed and oriented structures can show birefringent properties, looser more random arrangements would not show such birefringence. Many of the fibrils traversed the tubule wall in both a left handed and right handed spiral fashion, and probably interwoven with those from neighbouring tubules. Opposing spirals appeared to be separated by angles of $50-65^{\circ}$. The TEM images shown by Chen and Broom (1998) can also be interpreted as showing a similar arrangement, albeit following the disruption caused by chemical fixation. When studied with an FESEM, what appeared to be smaller fibrils of approximately $10 \mathrm{~nm}$ diameter were resolved, arranged orthogonally to the radial collagen fibrils, but only around the inside walls of the tubules. The spacing between these orthogonal fibrils was regular, between $60-70 \mathrm{~nm}$ (Figure 7). These fibrils, or their remnants, could also be seen in TEM sections of microwave-assisted fixed and $\mathrm{pH} 8.0$ fixed material (Figures 8 and 9), as well as close to the calcified / radial zone interface in those samples fixed chemically at pH8.0 (Figure 10). Fixation in a standard $2.5 \%$ glutaraldehyde at $\mathrm{pH} 7.0$ resulted in a complete breakdown of the structure, as seen by SEM (Figure 11) and TEM (Figure 15).

Cross-sectional fractures through the radial zone revealed a distinct regularity to the observed pattern of tubules and chondrons (Figure 12). Similarly, in some longitudinal fractures of the region - especially those fixed chemically - the chondrons appeared to be wholly enclosed within the columns of collagen fibres, and therefore not visible in the radial fractures (Figure 13). These observations suggested a role for these cells in the maintenance of the tubular structure. Computer-aided image analysis of such cross-sectional areas revealed that approximately $60 \%$ of the cross-sectional area of the tissue, at this point, was composed of tubular lumen. The average, minimum and maximum cross-sectional areas of the tubules were approximately 5,2 and $7 \mu \mathrm{m}^{2}$, respectively.

Why has such an organisation of collagen fibrils gone undiscovered in such an intensively studied tissue? The answer lies, we believe, in the fundamental nature of the tissue itself and the histological preparation methods that have normally been used to study it. The tubular organisation of collagen fibrils was initially observed only after AC had been subjected to cryo-fixation followed by freezesubstitution. Hunziker (1993), shows that AC collagen matrix structure is prone to significant modifications as a result of treatment with conventional chemical fixation procedures. Minor modifications of chemical fixation protocols can produce significantly different AC morphology. Rabbit AC shrinks considerably following chemical fixation and dehydration (Kääb et al., 1999b). The collagen-based structure was clearly disturbed by chemical fixatives, however at $\mathrm{pH} 8.0$ fixation some elements of the tubular structure were preserved, although still disrupted (Figures 9 and 14). Comparatively, freeze-substitution resulted in minimal disruption of the tissue, although some element of ice-crystal formed artefacts could have been expected - but the size of the crystals formed within the aggrecan contents of the collagen bound tubules appeared to be relatively small (Figure 16).

In spite of being regarded generally as effective fixatives of biological tissues, aldehydes are known significantly to reduce the number of positive charges on proteins (Hopwood, 1972) and can cause severe disruption of cytoplasmic structural proteins (Hayat, 1987). The balance of negative and positive charges on closely apposed fibrils and aggrecan assemblies could play a key role in the tight packing of the collagen matrix, as well as the formation of the general ultrastructure of the tissue, in vivo. Creating an electrostatic imbalance, by adding the fixing agent, would probably cause the structure to disassemble. If the walls were destabilised in this way the positive osmotic pressure of 2atm or more, in the PG rich tubule contents, would effect an influx of water on a large scale, resulting in disruption of the tubular morphology. Subsequent cross-linking of the PGs to each other and to collagen, by aldehyde fixatives, could then stabilize the modified microstructure. The appearance of chemically fixed and fractured tissue seemed to confirm that interpretation (Figure 11). Dehydration would then cause shrinkage of the tissue. This would result in compaction of the already disrupted fibrils, giving the appearance of a sponge-like tissue with some radial orientation - precisely that observed upon the examination of TEM images of conventionally prepared tissue (Figure 15). There was no suggestion of the level of organization observed after freeze-substitution (Figures 5 to 7 ), but 


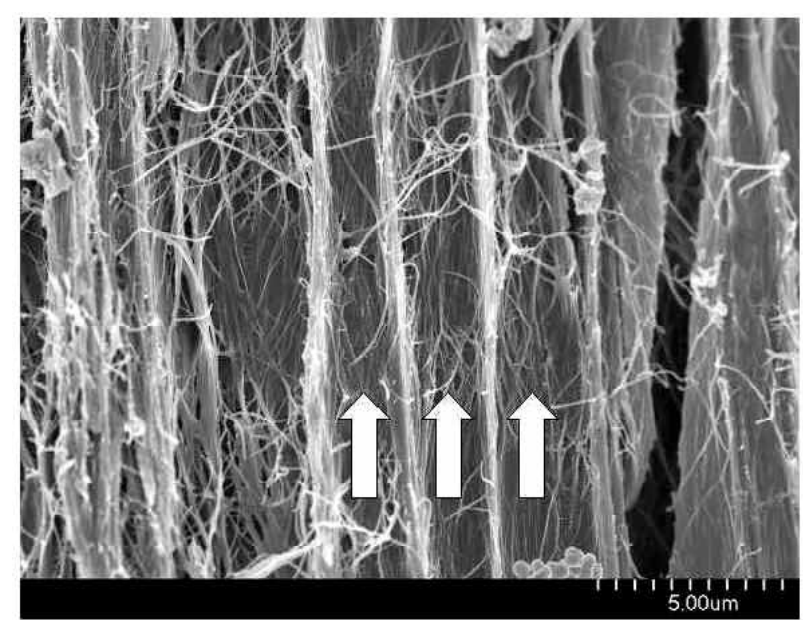

Figure 5: Medium power FESEM image showing the tubules in a longitudinal fracture of the tissue from the radial zone. Many of these tubules (arrows) were traced and found to be continuous from the calcified to the superficial zone.

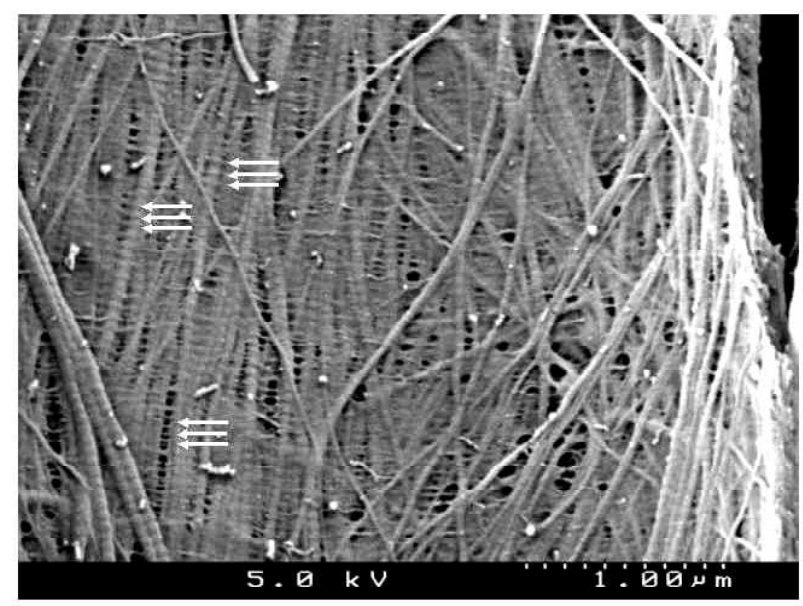

Figure 7: High power FESEM image showing the orthogonal array arrangement of the fibrils in a longitudinal fracture of a tubule from the radial zone. The $10 \mathrm{~nm}$ circumferential fibrils were regularly spaced (arrows), overlying the $30 \mathrm{~nm}$ radial fibrils, in spite of the disruption caused to the tissue by the fracturing procedure. Occasional spirally arranged fibrils were also seen on the inside of the lumen.

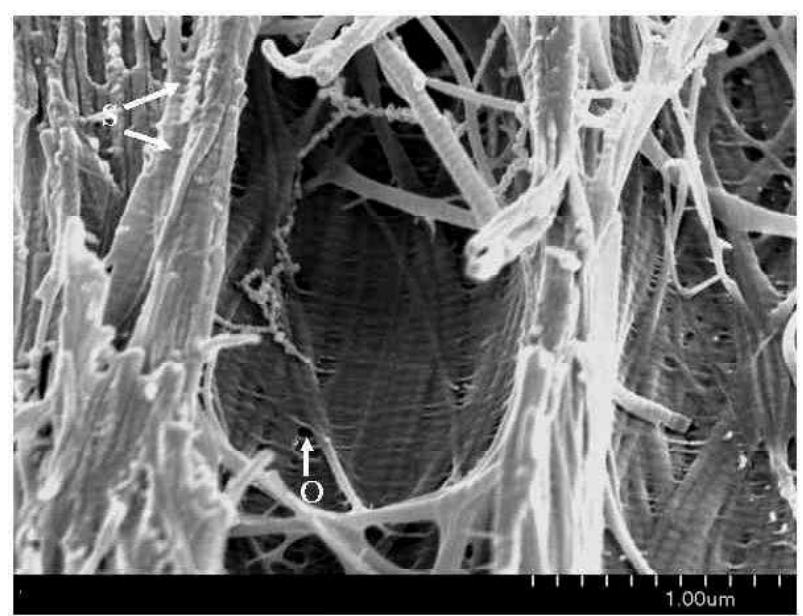

Figure 6: High power FESEM image of a longitudinal fracture of an individual tubule, showing the orthogonal array arrangement of the small fibrils. The $10 \mathrm{~nm}$ fibrils were regularly spaced $(\mathrm{O})$, overlying the $30 \mathrm{~nm}$ radial fibrils, in spite of the disruption caused to the tissue by the fracturing procedure. The spiral arrangement of fibrils in the tubule wall can also be seen (S).

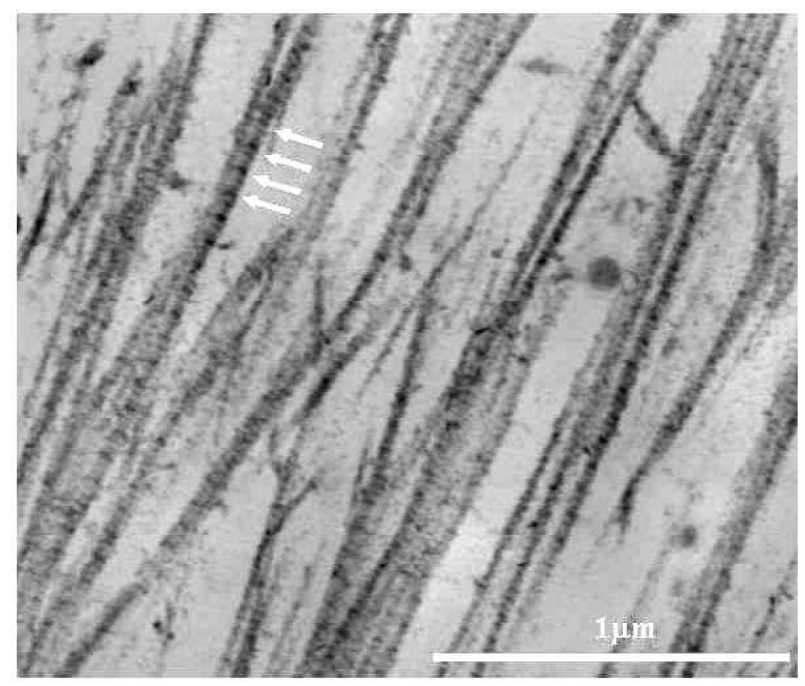

Figure 8: TEM image of microwave-assisted chemical fixed radial zone articular cartilage tissue. Some of the $30 \mathrm{~nm}$ radial fibrils remained attached to each other in bunches and $10 \mathrm{~nm}$ fibrils appear bound to those bunches at $60-70 \mathrm{~nm}$ intervals (arrows). microwave-assisted chemically fixed preparations did retain a certain element of the original structure (Figure 8). Microwave-assisted chemical fixation is known to be less damaging to AC tissue than unassisted chemical fixation, although exactly why this should be so is not really understood (Richards and Kääb, 1996; Kääb et al., 1999b). When conventional fixation was carried out at $\mathrm{pH} 8.0$ a certain amount of the tubular morphology was retained within the tissue, including some of the orthogonal fibril arrangement (Figures 9 and 10), especially close to the radial zone / calcified zone interface. This confirmed that the tubular structures, initially observed following the application of freeze substitution protocols alone, appear to be genuine and not artefacts of the specimen preparation procedure.

It is also significant that in the freeze-substituted preparations that there was only visual evidence of ice crystal formation within the tubule lumen contents and none in the walls, suggesting a very low water content in that area (Figure 16). The behaviour of the collagen matrix in response to chemical fixatives could, therefore, be providing us with valuable evidence as to the way in which that matrix is normally held together, electrostatic charge balance being implicated. It is also significant that AC tissue fixed chemically was relatively easily impregnated with embedding resin, whereas the freeze-substituted tis- 


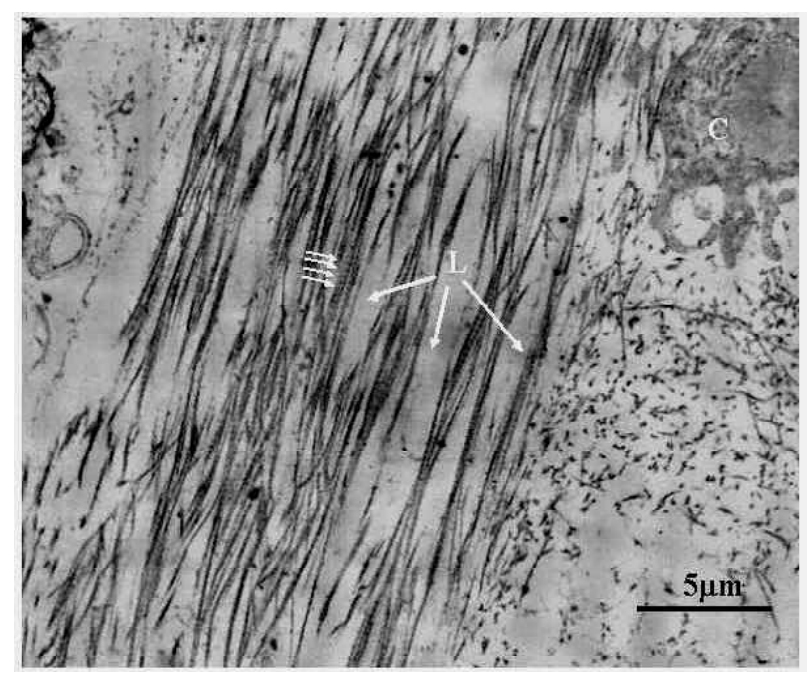

Figure 9: TEM image of chemically fixed radial zone articular cartilage tissue. In this case $2.5 \%$ glutaraldehyde, buffered at $\mathrm{pH} 8.0$, with $0.5 \mathrm{M}$ sucrose, for 2 hours at $20^{\circ} \mathrm{C}$. Some of the $30 \mathrm{~nm}$ radial fibrils remained attached to each other in bunches and $10 \mathrm{~nm}$ fibrils appear bound to those bunches at $60-70 \mathrm{~nm}$ intervals (arrows). The lumen of the tubules (L) appears to have been preserved to a certain degree. The chondrocyte $(\mathrm{C})$ in the neighbouring chondron is also seen.

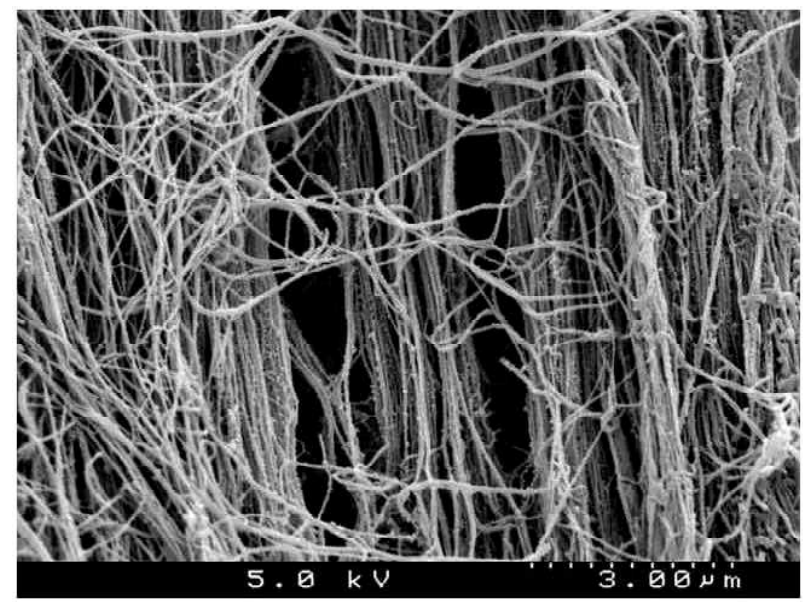

Figure 11: FESEM image of a longitudinal fracture of the radial zone, this tissue had been fixed for 2 hours in $2.5 \%$ glutaraldehyde at $\mathrm{pH}$ 8.0. Following dehydration and critical point drying the sample was fractured at liquid nitrogen temperature before returning to room temperature in a dry environment. Severe disruption of the collagen matrix was evident.

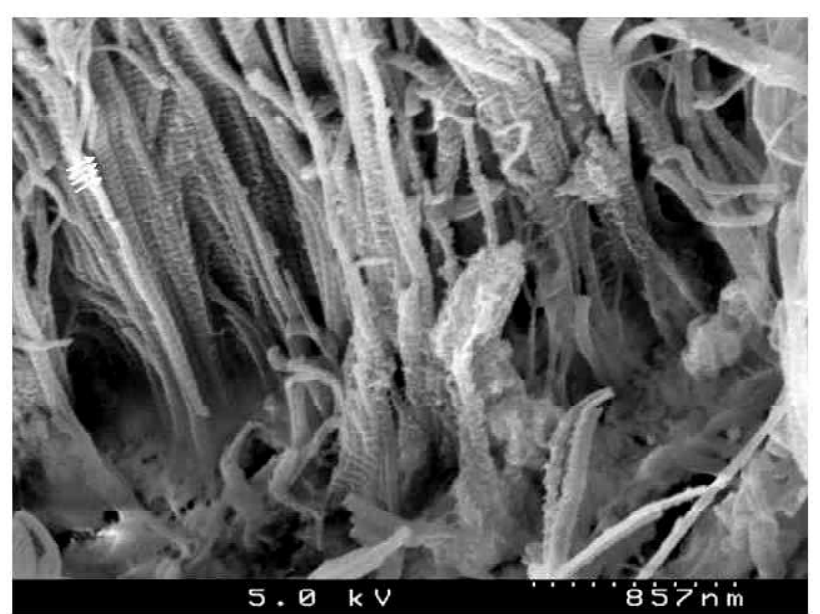

Figure 10: FESEM image of adult rabbit tibial articular cartilage, fixed at $20^{\circ} \mathrm{C}$ in $2 \%$ acrolein buffered in McIlvaine's buffer at pH8.0 (Bancroft and Cook, 1994). The image shows the base of the radial zone, at the interface with the calcified zone. Bases of tubular structures are seen, including orthogonally arranged fibrils apparently binding the $30 \mathrm{~nm}$ diameter collagen fibrils lining the tubules' walls (arrows). More than a few microns from the interface the collagen structure was entirely disrupted.

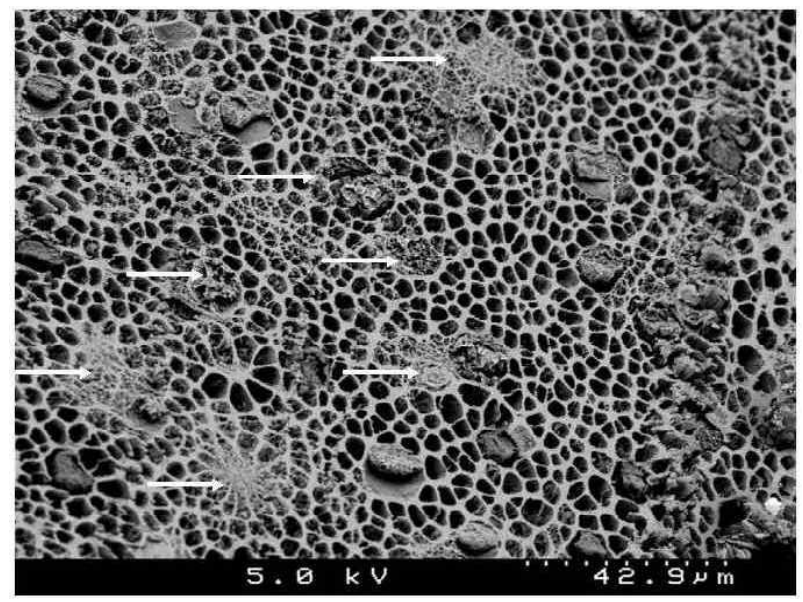

Figure 12: Low magnification FESEM image of a cross fracture of the radial zone, close to the calcified layer, showing the tubules formed by the segregation of the tightly packed collagen fibrils. Cross-sections of the chondrons (arrows) were regularly spaced throughout the tissue. The diameters of most tubules were in the range of $1-3 \mu \mathrm{m}$. sue was extremely difficult to embed - requiring resin impregnation times of several weeks, even with a very low viscosity resin. This again suggested that there was a fundamental structural difference between the results of applying these diverse preparation techniques to $\mathrm{AC}$, and that chemical fixation disrupted severely the natural arrangement of the tissue components. This confirmed what was observed by means of microscopy.

Freeze-substitution appeared to be the only approach capable of coming close to preserving the internal morphology of this tissue for study by SEM, FESEM or TEM.
Kellenberger (1990) and Edelmann (1994) argue that the application of freeze-substitution results in the production of fewer ultrastructural artefacts than do conventional chemical fixation techniques, provided reasonably rapid freezing can be achieved. A comparison of the effects of applying different preparation methods to the preservation of AC emphasises the advantages of using freezesubstitution, especially in relation to the reduction of tissue shrinkage (Kääb et al., 1999b).

There are limitations to the cryo-fixation approach, one of them is the problem of ice crystal formation, if 


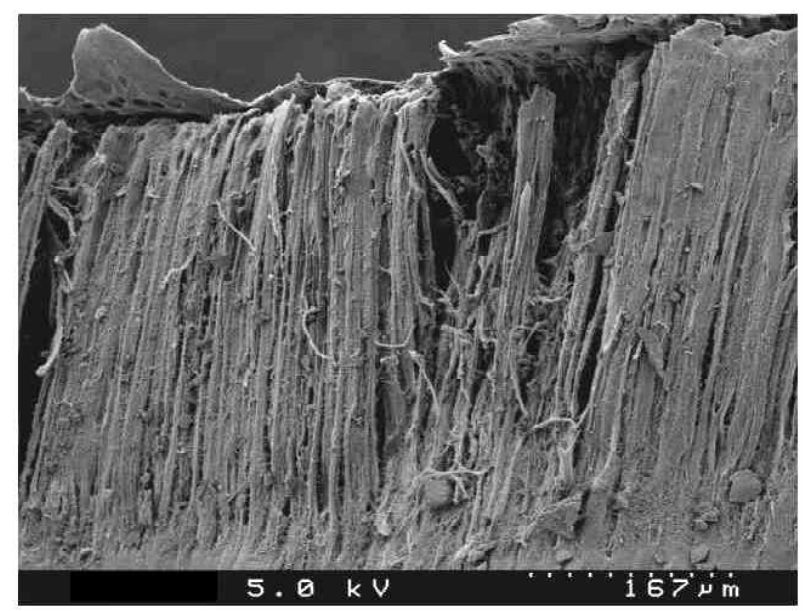

Figure 13: Low magnification FESEM image of columns of fibres in adult rabbit tibial articular cartilage fixed chemically in 2\% glutaraldehyde in McIlvaine's buffer ( $\mathrm{pH}$ 8.0), dehydrated, critical point dried and fractured at liquid nitrogen temperature. Even at this magnification the disruption caused by chemical fixation was evident and the chondrons appeared to be enclosed in the matrix, and not exposed in the fracturing process.

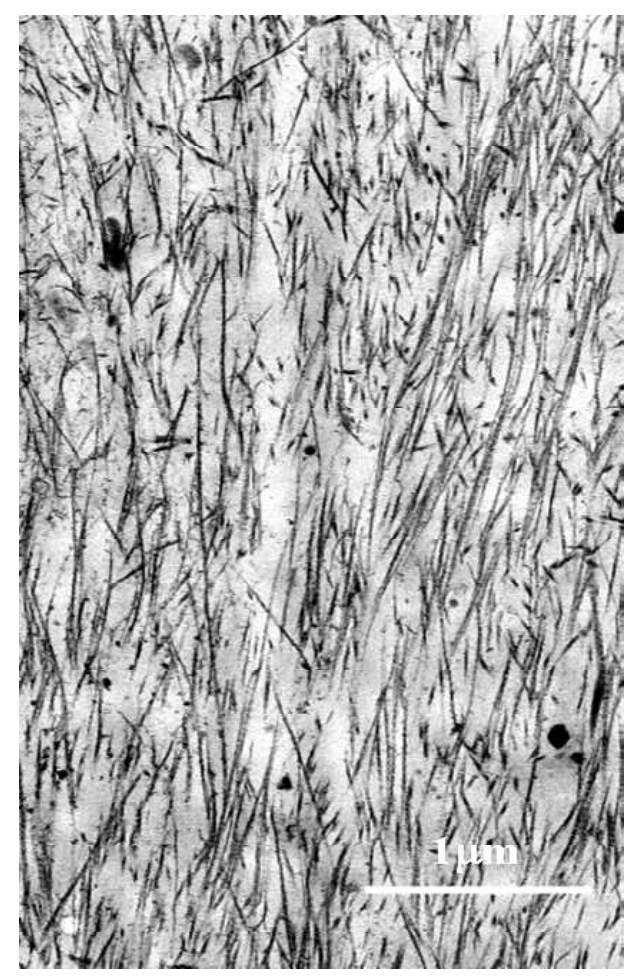

Figure 15: TEM image of an ultrathin section of a chemically fixed sample of radial zone articular cartilage tissue. Practically all of the lateral association of the radial $30 \mathrm{~nm}$ collagen fibrils was disrupted, although the general radial orientation was retained. This is the hitherto perceived arrangement of collagen fibrils in the radial zone of this tissue.

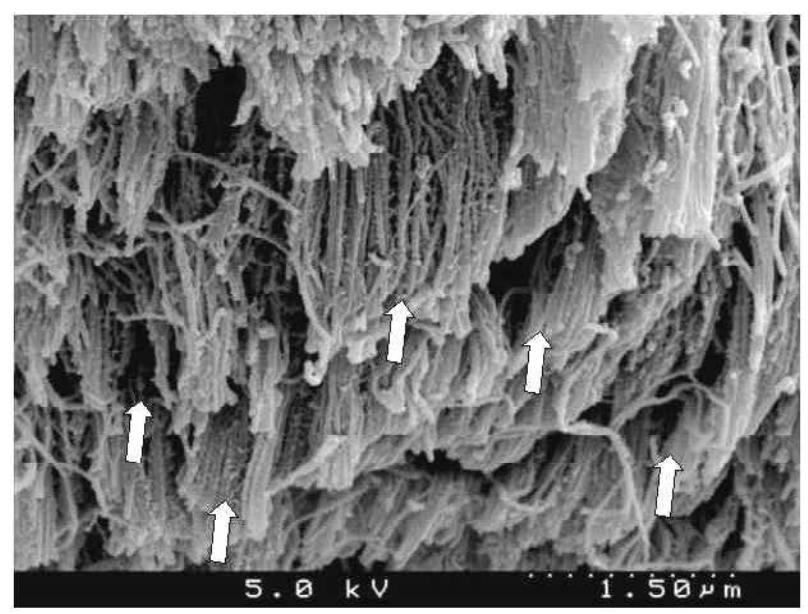

Figure 14: FESEM image of a cross-fracture of a radial zone column of adult rabbit tibial articular cartilage fixed chemically in $2 \%$ acrolein in McIlvaine's buffer ( $\mathrm{pH} 8.0$ ), dehydrated, critical point dried and fractured at liquid nitrogen temperature. Although the whole specimen had shrunk, the essentials of the tubular arrangement of collagen fibrils (arrows) were still present. The thin, orthogonally arranged fibrils, appeared to have been broken - but fragments of them remained attached to the main radial fibrils.

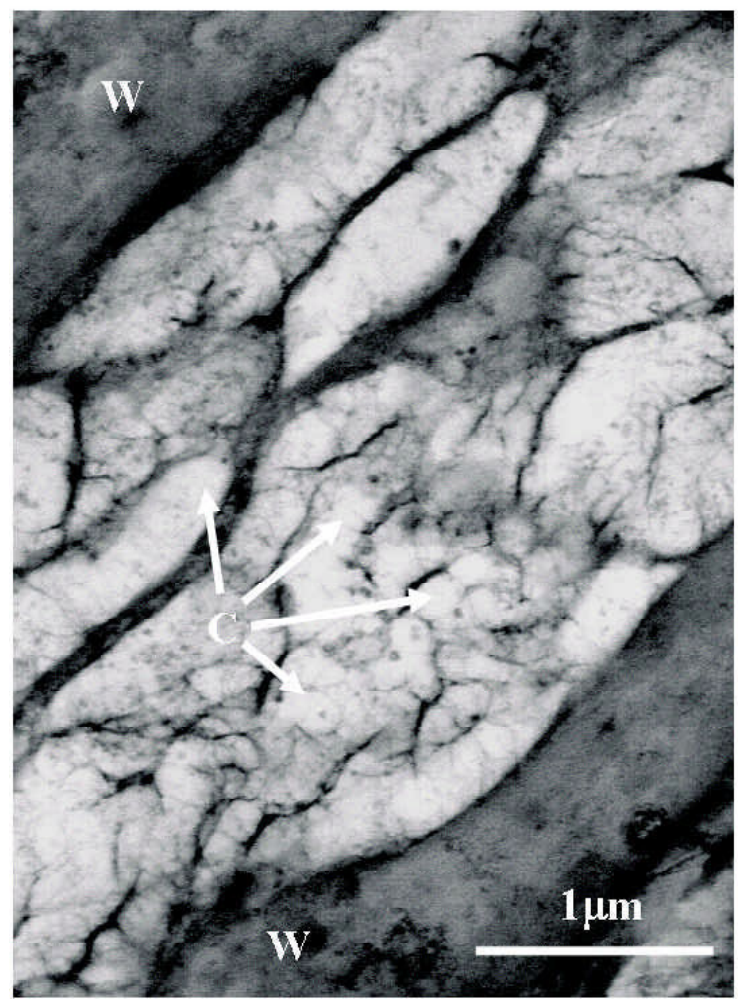

Figure 16: TEM of freeze-substituted and embedded rabbit tibial articular cartilage. Close lateral packing of the radial $30 \mathrm{~nm}$ collagen fibrils was retained, as shown in the FESEM images, forming the tubule walls (W). Within the tubule lumen the proteoglycans were segregated, during the freezing process, due to the formation of ice crystals (C). The sizes of the ice crystals formed were much smaller than the cross sectional diameter of the tubules. There was no visible evidence for the formation of ice crystals within the tubule wall structure (W). 


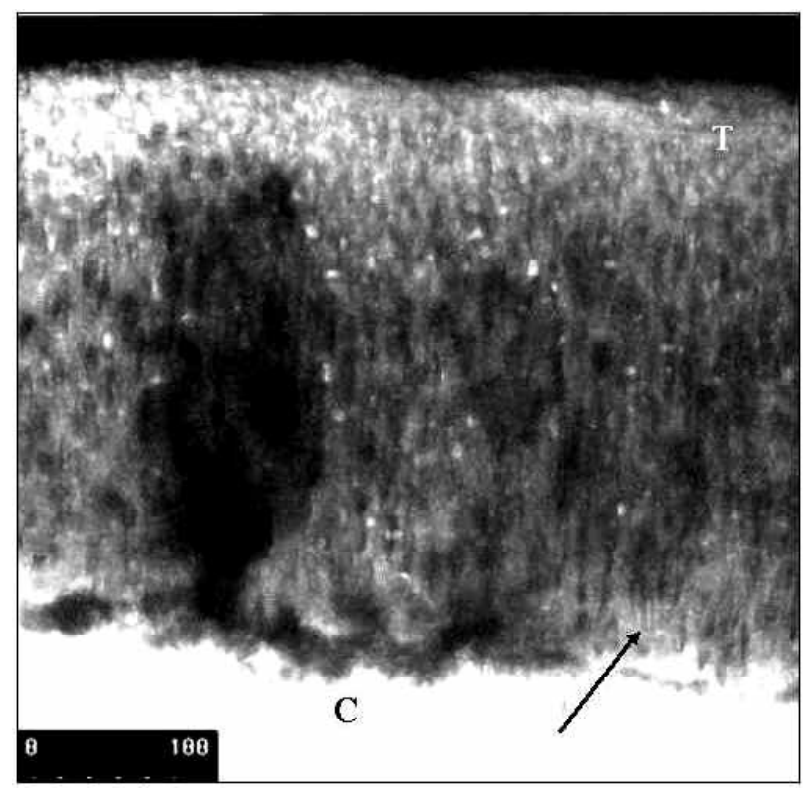

Figure 17: Confocal laser scanning microscope picture of freshly cut rabbit tibial articular cartilage from the knee joint. Contrast was generated by the autofluorescence of collagen when illuminated by light at $488 \mathrm{~nm}$ wavelength. Evidence for the presence of 1$3 \mu \mathrm{m}$ tubular structures, from which collagen was excluded, was seen in the radial zone of the tissue (arrow). Superficial zone morphology was distinctly different $(\mathrm{T})$. The whole depth of tissue from calcified zone $(\mathrm{C})$ to the superficial zone is shown.

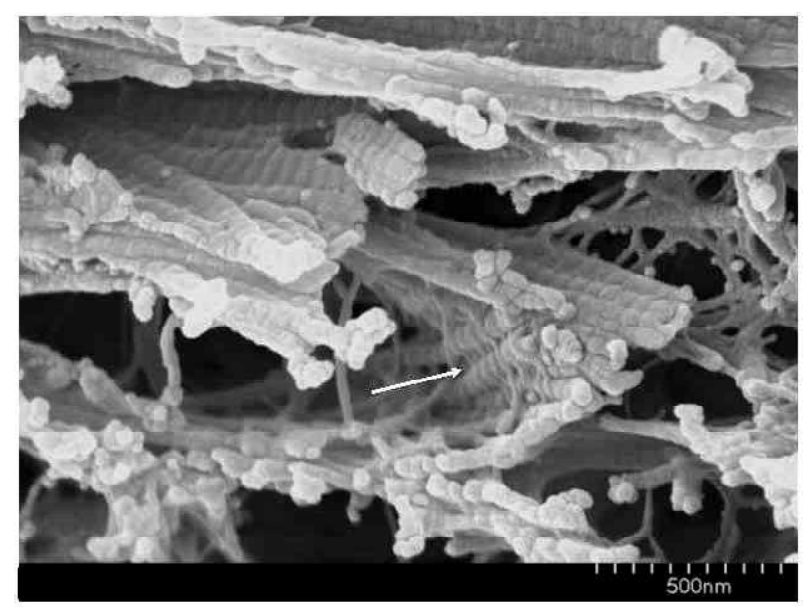

Figure 18: High power FESEM image of a longitudinal fracture of part of the superficial zone, showing the orthogonal array arrangement of the small fibrils. The $10 \mathrm{~nm}$ fibrils were regularly spaced (arrow), overlying the $30 \mathrm{~nm}$ radial fibrils, in spite of the disruption caused to the tissue by the fracturing procedure.

freezing is carried out too slowly or the specimen is allowed to warm up above the recrystallisation point for its contents (generally about $-70^{\circ} \mathrm{C}$ ). This can happen if the specimen is too large or there is a large amount of 'free' water available for crystal formation. Ice crystals can damage the ultrastructure of tissues because they occupy a larger volume than the fluid water from which they are formed and their formation removes water from the sur- rounding material thereby increasing the concentration of solutes. Very rapid freezing is necessary to cool the tissue below the re-crystallisation point of water, in as short a time as possible, and therefore minimise these effects (Echlin, 1992). High-pressure freezing is the preferred method by which optimal freezing rates are obtained, as long as the specimen size does not exceed $200 \mu \mathrm{m}$ in diameter (Studer et al., 1995). This means that the tissue needs to be cut into very small pieces before freezing, which in the case of AC is almost certain to disrupt its overall structure. Placing an excised sample of AC, when separated from the calcified zone, into a buffer resulted in considerable swelling of the tissue. Interpretation of the overall morphology of AC in samples of this size would also be difficult without prior knowledge of the structure at a more intermediate level. Chemical preparation methods may not even preserve the ultrastructure of those larger specimens in a way necessary to elucidate their morphology at such magnifications. The only approach available was to optimise the freezing procedure, as applied to cores of AC that were complete from articulating surface to the calcified zone. Such samples were too large for high-pressure freezing. The fastest freezing method available was rapid plunging into liquid propane at $-196^{\circ} \mathrm{C}$.

The possibility of the observed tubules being artefacts of ice crystal formation, as a result of the application of conditions that would not be expected to lead to vitrification, has already been discounted (ap Gwynn et al., 2000). Although small ice crystals are observed, the tubular arrangement of collagen fibrils is genuine. This was confirmed by their appearance following the application of modified chemical fixation procedures, where there was no possibility of ice-crystal formation taking place (Figure 14). An image of freshly cut tissue, which had not been frozen, taken by confocal laser scanning microscopy utilising the auto-fluorescent property of collagen at $488 \mathrm{~nm}$, also provided evidence for the presence of very narrow, vertically oriented, areas within the radial zone of the tissue from which collagen was excluded (Figure 17). Chondrons were also seen as areas of collagen exclusion, but were larger.

Studer et al. (1995) demonstrate that PG can act as a natural cryoprotectant in AC. They show that, in bovine $\mathrm{AC}$, vitrification of the tissue, during rapid cooling, is dependent on water content and solute concentration. When AC is high-pressure frozen, the superficial-transitional zone and upper radial zone, which are closer to the surface, cryo-fix in a crystalline state but the deep radial zone is fully vitrified. The superficial and upper radial zones have a water content of $75-80 \%$ and a proteoglycan content of $0.5-2 \%$ and $2-4 \%$ respectively, the deep radial zone has a water content of $65 \%$ and a proteoglycan content of $4-8 \%$. Ice crystal growth in the superficial zone is so small that only segregation artefacts of the proteoglycans are observed. It is unlikely therefore that significant ice crystal growth could occur in the deep radial zone, even if plunge freezing in liquid propane was used rather than high-pressure freezing.

Type IX collagen has the ability to bind to the surface of type II collagen and also to other type IX collagen molecules. It, therefore, has the potential to form covalent 
links between type II fibrils (Eyre and Wu, 1995). Type IX collagen binds to some type II fibrils with a D-periodic spacing of about $65 \mathrm{~nm}$, in material from human infant AC (Bruckner et al., 1988). A similar D-periodic binding has been observed in chick embryo sternal and bovine articular cartilage (Eyre et al., 1987; van der Rest and Mayne, 1988; Vaughan et al., 1988). This is very similar to the spacing we observed between the orthogonally arranged $10 \mathrm{~nm}$ fibrils and the $30 \mathrm{~nm}$ radial fibrils on the inside of the tubes (Figures 6,7 and 8).

Type XI collagen can also form fibrils. The material that appeared to bind orthogonally at regular $65 \mathrm{~nm}$ spacing to the radial collagen fibrils in both the high resolution FESEM images of freeze substituted samples (Figure 7), chemically fixed material (Figures 9 and 10) and the TEM images of microwave-assisted fixed material (Figure 8), may well have represented where components such as collagen IX and/or XI bound the collagen II fibrils together in the tightly-bound network of fibrils that made up the inner 'surface' wall of the tubules - also possibly linking to the PGs in the lumen of the tubule. The evidence for this interpretation is circumstantial but the dimensions, and binding pattern, of the fibrils did correspond to those expected of these collagen types. The stability of this complex may, in itself, depend upon the integrity of the tissue. The attraction of ions and water to the PG in the tubules may have provided a stabilising mechanism that maintained the tightly packed structure of the tubule walls. An orthogonal array, only stable in a certain range of collagen II, IX, XI and PG concentrations (and any other components that may be present), may have provided the structure with some stiffness to resist compressive load in equilibrium. It may do this by promoting the binding of collagen II fibrils together. It is known that type XI collagen forms heterotypic fibrils with type II collagen (Bland and Ashurst, 1996). In the superficial zone, there was a smaller diameter tubular morphology, arranged in a direction parallel to the articulating surface, but not appearing to be formed into complete tubules. Small areas of orthogonally arranged small fibrils were also seen in this zone (Figure 18). It was the deeper radial zone that appeared to have the most well organised and thicker tubular walls, corresponding to the region where the PG concentration is believed to be highest (Studer et al., 1996). Their regular spacing (Figure 12), position and columnar arrangement suggested that the cells in the chondrons might also play a key role in the maintenance of the tubular structure.

It is interesting to note that type IX collagen does not bind to all fibrils from the same digest and that it may prevent the formation of large fibres of type II collagen by connecting thin fibrils (Bruckner et al., 1988), in which case it seems reasonable to assume that all fibrils would be involved. A similar question arises in its proposed role, with proteins such as decorin, to shield fibrils from PGs to reduce friction between fibrils and non-fibrillar components of AC (Studer et al., 1996). These observations, and the relative proportions of these proteins in the tissue, are consistent with the type IX collagen being a component of the interface between PG and collagen lin- ing the lumen of the tubules. Such an orthogonal array of fibrils, as was seen in the relationship between the radial and orthogonally arranged fibrils in the tubule walls, is not a unique biological structure. A similar structure is found in the penis of Dasypus novemcinctus (Kelly, 1997), where resistance to compression is also achieved. Similar pleating to that observed for heavily loaded AC (Kääb et al., 1999a) is also seen in the collagen-based structures of the penis when it is flaccid.

Double or triple layered, opposed, helical arrangement of collagen fibrils is common in invertebrates with hydrostatic skeletons (Harris and Crofton, 1957). In such cases the arrangement of the collagen fibrils can be shown to resist longitudinal deformation of the opposed helical structure, when held in a contained system. The critical factor is the angle between the opposing helices. As the value for this angle increased, as would happen if the tissue was compressed longitudinally, then the hydrostatic pressure within the contained volume would increase correspondingly. This is how nematode worms are able to dispense with the need for circular muscles, and are able to move with only longitudinal muscles. The force to return the animal to its full length is achieved solely due to the arrangement of the collagen fibrils in its cuticle. It is possible that the physical arrangement of the collagen fibril matrix in the AC radial zone could also make a contribution to compression resistance in the tissue, and especially its return to full thickness when a load is removed.

When AC is loaded, initially the superficial zone underneath the contact area is highly compressed. With time the fluid redistributes radially, distributing the strain uniformly. The strain then decreases in the more superficial layers and increases in the deeper layers. In addition, some fluid continues to exude from the $\mathrm{AC}$ region beneath the contact area allowing further depression of the AC surface. At equilibrium, the tubular structure of collagen probably does not enhance the stiffness of AC because the increased concentration of PGs, with swelling pressures greater than $2 \mathrm{~atm}$, would be sufficient to support the measured aggregate modulus of 0.5 to $2 \mathrm{MPa}$. However during the transient response, we propose that the tubular collagen structure inhibits lateral fluid flow, parallel to the surface, forcing fluid to flow perpendicular towards the surface. Without being able to escape laterally into a more permeable region of AC, the fluid would have to flow within the tubules through less permeable compressed PGs and through the highly compressed dense layer of fibrils in the superficial zone. This mechanism would be optimal for AC to hold larger loads for longer durations without significant, potentially damaging, deformation of its solid matrix.

Although this suggested mechanism is still only an hypothesis developed from newly exhibited collagen-fibril based structure, there are several observations and studies in the literature that would support such an idea. Kääb et al. (1999a) studied the collagen morphology of ex vivo physiologically loaded rabbit tibial cartilage. They show that even under $3 \mathrm{x}$ body weight load at the knee, the AC collagen matrix directly adjacent to that beneath 
the loaded contact area is hardly deformed and does not bulge laterally. This lack of adjacent matrix deformation may exhibit the extent to which the collagen tubules support the fluid pressure radially with respect to the tubular axis.

Macirowski et al. (1994), combining an elegant experimental and numerical modelling approach, examined the interstitial fluid flow and pressurisation within acetabular AC. They found that even after $20 \mathrm{~min}$ of loading, fluid continues to support $90 \%$ of the applied load. The resistance to fluid flow is much higher parallel to the surface than in the perpendicular direction, perhaps due to fluid channelling by the tubules. The fluid flow is predominantly in the perpendicular direction, and the consolidation rate is controlled by conductance of the interarticular gap. Because the soft superficial zone could easily seal this gap, the AC is able to maintain fluid pressurisation for an extended duration. This observation is confirmed by Soltz and Ateshian (1998). Finally, Setton et al. (1993) tested bovine osteochondral plugs in confined compression, with and without the superficial layer. They find that without the superficial layers the permeability of the tissue increases almost 2-fold with increased early creep rates but that there is no change in the equilibrium modulus. Such a change would be consistent with a channelling of fluid flow within the tubules where the fluid flowing, predominantly perpendicular to the surface, would need to pass through the compressed highly impermeable superficial zone, but that the osmotic swelling pressure of the compressed PGs within the tubules remains unchanged.

Does rabbit AC structure differ from that of human? There may be very little difference between the fundamental structure of this tissue in different species (Zambrano et al., 1982; Clark, 1991). A similar tubular structure should therefore be expected in all mammals. Studies of gross morphological differences between species suggest a categorisation into either a column-based (often referred to as 'fibre-based') or a leaf-based overall arrangement (Clark, 1991; Teshima et al., 1995; Kääb et al., 1998). The fundamental tubular arrangement proposed would be expected to occur within those columnar or leaf structures, and preliminary studies of tissue from other species, including human, suggested that was probably the case. Initial investigations also suggested that a variation upon the pattern also exists in the load bearing areas of the rabbit hip and elbow joints.

\section{Conclusions}

We have provided further evidence to show that the collagen matrix in the radial zone of rabbit tibial AC was arranged into radially arranged tubules. Both chemical and cryo-specimen preparation techniques were shown to preserve this structure. The arrangement of collagen fibrils in the tubule wall would suggest a structure evolved to contain and or direct the flow of more fluid contents in a direction perpendicular to the articulating surface, and in itself also possibly provide some resistance to compression. The structure we described was consistent with the known physical, chemical and structural evidence available to describe the tissue, but raised many questions that need answering. How could such a tissue develop from early embryonic limb structures through to adult cartilage? Is the collagen laid down in a similar way to that found in fibro-cartilage tendon development? How are the tubular collagen/PG structures formed initially? Is physical loading necessary for the structure to form? Does the structure break down in cases of OA and how does superficial layer damage effect the progression of OA? Can the tubular structure be induced to grow and re-form in mature adult tissue, if it has been damaged? Work is currently in progress in an attempt to answer some of these questions.

\section{Acknowledgements}

We would like to thank the following for their assistance during the course of this work: I AlAmry, CW Archer, M Capers, H and I Gerber, MJ Kääb, PC Lloyd, P Monaghan, M Müller, GRh Owen, A Pugh, S Turner, D Williams. The AO Research Fund (AORF), Dubendorf, provided financial support for this project (Grant numbers $98 \mathrm{G} 36$ and 2000G50).

\section{References}

ap Gwynn I, Wade S, Kääb MJ, Owen GRh, Richards RG (2000) Freeze-substitution of rabbit tibial articular cartilage reveals that radial zone collagen fibres are tubules. J Microsc 197: 159-172.

Basser PJ, Schneiderman R, Bank RA, Wachtel E, Maroudas A (1998) Mechanical properties of the collagen network in human articular cartilage as measured by osmotic stress techniques. Arch Biochem Biophys 351: 207-219.

Bayliss MT, Howat S, Davidson C, Dudhia J (2000) The organization of aggrecan in human articular cartilage - Evidence for age-related changes in the rate of aggregation of newly synthesized molecules. J Biol Chem 275: 6321-6327.

Benninghoff, A (1925) Form und Bau der Gelenkknörpel in ihren Beziehungen zur Funktion (Form and structure of articular cartilage in their relation to function). Z Zellforsch 2: 783-862.

Bland Y S, Ashurst D E (1996) Development and ageing of the articular cartilage of the rabbit knee joint: distribution of the fibrillar collagens. Anat Embryol 194: 607-619.

Broom ND (1986) The collagenous architecture of articular cartilage - A synthesis of ultrastructure and mechanical function. J Rheumatol 13: 142-150.

Bruckner P, Mendler M, Steinmann B, Huber S, Winterhalter K (1988) Structure of human collagen type IX and its organization in fetal and infant cartilage fibrils. J Biol Chem 263: 16911-16917.

Buckwalter JA, Rosenberg LC, Ungar R (1987) Changes in proteoglycan aggregates during cartilage mineralization. Calcif Tiss Int 41: 228-236.

Chen MH, Broom N (1998) On the ultrastructure of softened cartilage: a possible model for structural trans- 
formation. J Anat 192: 329-341.

Clark JM (1991) Variation of collagen fibre alignment in a joint surface - a scanning electron-microscope study of the tibial plateau in dog, rabbit and man. J Orthop Res 9: $246-257$.

Clarke IC (1971) Articular cartilage: a review an electron microscope study. J Bone Joint Surg 53B: 732-750.

Echlin P (1992) Low-Temperature Microscopy and Analysis. Plenum, New York and London. pp 19-57.

Edelmann L (1994) Low temperature embedding of chemically unfixed biological material after cryosorption freeze-drying. Scanning Microsc 8: 551-562.

Eyre DR (2001) Collagen of articular cartilage. Arthritis Res 4: 30-35.

Eyre DR, Wu JJ (1995) Collagen structure and cartilage matrix integrity. J Rheumatol 22: 82-85.

Eyre DR, Wu JJ, Apone S (1987) A growing family of collagens in articular cartilage: identification of 5 genetically distinct types. J Rheumatol 14: 25-27.

Harris JE, Crofton HD (1957) Structure and function in the nematodes: Internal pressure and cuticular structure in Ascaris. J Exp Biol 34: 116-130.

Hayat M A (1987) Principles and Techniques of Electron Microscopy. 3rd Edition, MacMillan, London. pp 1314.

Hopwood D (1972) Theoretical and practical aspects of glutaraldehyde fixation. A review. Histochem J 4: 267 303.

Hunziker EB (1993) Application of cryotechniques in cartilage tissue preservation and immunoelectron microscopy: potentials and problems. Microsc Res Tech 24: 457-464.

Hunziker EB, Wagner J, Studer D (1996) Vitrified articular cartilage reveals novel ultrastructural features respecting extracellular matrix architecture. Histochem Cell Biol 106: 375-382.

Hunziker EB, Michel M, Studer D (1997) Ultrastructure of adult human articular cartilage matrix after cytochemical processing. Microsc Res Techn 37: 271-284.

Jeffery AK, Blunn GW, Archer CW, Bentley G (1991) 3-dimensional collagen architecture in bovine articularcartilage. J Bone Joint Surg 73B: 795-801.

Kääb MJ, ap Gwynn I, Nötzli HP (1998) Collagen fibre arrangement in the tibial plateau articular cartilage of man and other mammalian species. J Anat 193: 23-34.

Kääb MJ, Ito K, Clark JM, Nötzli HP (1999a) Deformation of articular cartilage collagen structure under static and cyclic loading. J. Orthop Res 16: 743-751.

Kääb MJ, Richards RG, Walther P, ap Gwynn I, Nötzli HP (1999b) A comparison of four preparation methods for the morphological study of articular cartilage for scanning electron microscopy. Scanning Microsc 13: 61-70.

Kellenberger E (1990) The potential of cryofixation and freeze-substitution: observations and theoretical considerations. J Microsc 161: 183-203.

Kelly DA (1997) Axial orthogonal fiber reinforcement in the penis of the nine-banded armadillo (Dasypus novemcinctus). J Morphol 233: 249-255.

Lane M, Weiss C (1975) Review of articular cartilage collagen research. Arthr Rheum. 18: 553-562.
Macirowski T, Tepic S, Mann RW (1994) Cartilage stresses in the human hip joint. J Biomech Eng 116: 1018.

Morrison EH, Ferguson MW, Bayliss MT, Archer C (1996) The development of articular cartilage: 1. The spatial and temporal patterns of collagen types. J Anat 189: 9-22.

Nötzli H, Clark J (1997) Deformation of loaded articular cartilage prepared for scanning electron microscopy with rapid freezing and freeze-substitution fixation. $\mathrm{J}$ Orthop Res 15: 76-86.

Poole CA, Flint MH, Beaumont BW (1987) Chondrons in cartilage: Ultrastructural analysis of the pericellular microenvironment in adult human articular cartilage. J Orthop Res 5: 509-522.

Poole AR, Pidous I, Reiner A, Rosenberg LC (1982) An immunoelectron microscope study of the organization of proteoglycan monomer link protein and collagen in the matrix of articular cartilage. J Cell Biol 93: 921937.

Ratcliffe A, Mow VC (1996) Structure and function of articular cartilage. In: Extracellular Matrix (Comper WD, ed). Harwood Academic. pp 234-306.

Richards RG, Kääb MJ (1996) Microwave-enhanced fixation of rabbit articular cartilage. J Microsc 181: 269276.

Setton LA, Zhu W, Mow RW (1993) The biphasic poroviscoelastic behavior of articular cartilage: role of the surface zone in governing the compressive behavior. $\mathrm{J}$ Biomech 26: 581-592.

Soltz MA, Ateshian GA (1998) Experimental verification and theoretical prediction of cartilage interstitial fluid pressurisation at an impermeable contact interface in confined compression. J Biomech 31: 927-934.

Studer D, Chiquet M, Hunziker EB (1996) Evidence for a distinct water rich layer surrounding collagen fibrils in articular cartilage extracellular matrix. J Struct Biol 117: $81-85$.

Studer D, Michel M, Wholwend M, Hunziker EB, Buschbaum MD (1995) Vitrification of articular cartilage by high pressure freezing. J Microsc 179: 321-332.

Teshima R, Otsuka T, Takasu N, Yamagata N, Yamamoto K (1995) Structure of the most superficial layer of articular cartilage. J Bone Joint Surg 77B: 460-464.

Urban JP, Maroudas A, Bayliss MT, Dillon J (1979) Swelling pressures of proteoglycans at the concentrations found in cartilaginous tissues. Biorheology 16: 447-464.

van der Rest M, Mayne R (1988) Type IX collagen proteoglycan from cartilage is covalently cross-linked to type II collagen. J Biol Chem. 263: 1615-1618.

Vaughan L, Mendler M, Huber S, Bruckner P, Winterhalter KH., Irwin I, Mayne R (1988) D-periodic distribution of collagen type IX along cartilage fibrils. J Cell Biol 106: 991-997.

Williams JL, Vani JN, Eick JD, Petersen EC, Schmidt TL (1999) Shear strength of the physis varies with anatomic location and is a function of molecules, inclination and thickness. J Orthop Res 17: 214-222

Young RD, Lawrence PA, Duance VC, Aigner T, Monaghan P (1995) Immunolocalization of types III col- 
lagen in human articular cartilage prepared by high pressure cryofixation, freeze-substitution and low-temperature embedding. J Histochem Cytochem 43: 421-427.

Young RD, Lawrence PA, Duance VC, Aigner T, Monaghan P (2000) Immunolocalization of collagen types II and III in single fibrils of human articular cartilage. J Histochem Cytochem 48: 423-432.

Zambrano NZ, Montes GS, Shigihara KM, Sanchez EM, Junqueira LC (1982) Collagen arrangement in cartilages. Acta Anat 113: 26-38.

\section{Discussion with Reviewer}

C. Archer: How do the authors respond to the criticism that they are studying a freezing artefact?

Authors: To propose that these tubular structures that are observed in adult rabbit tissue are segregation artefacts is a perfectly natural reaction. Indeed, this was also our initial response. However, on further investigation it became clear that this was not the case.

By examining both TEM and SEM images, we could clearly see where small ice crystals had been formed. These were much smaller that the dimensions of the tubules. Without doubt, the formation of small ice crystals, within their lumens, would have caused the dimensions of the tubules to expand. This has clearly happened in some places - stretching the orthogonal fibrils. This does not mean that the tubules are formed by the segregation of larger ice crystals.

Much of the argument is dealt with, at some length, in our Journal of Microscopy paper (ap Gwynn et al., 2000). Here we show that the tubular structure is preserved even after the addition of cryoprotectants, and that the dimensions of the tubules remain relatively constant even when different freezing procedures are used. The dimensions also remain constant regardless of the depth into the tissue. If the structures were formed by ice crystals, then the areas further away from the tissue/coolant interface - subject to slower cooling rates - would be expected to show larger ice crystal artefacts. They do not show such differences. Studer et al. (1995) demonstrated that the concentration of PGs is much higher in the deeper zones of the tissue. This would be expected to provide an increasing cryo-protective effect at greater depth, resulting in smaller ice crystals.

In the current paper we have shown that the rudiments of the tubular structure are also preserved after the application of a much modified chemical fixation protocol where no freezing has been applied. It is difficult to imagine how freezing artefacts could be formed without the application of low temperature! Similarly, the essentials of the vertical segregation of the tissue components can be just resolved, with a confocal laser scanning microscope, in wet tissue - in this case depending upon the natural fluorescence of the collagen itself to form an image.

The clear presence of the orthogonally arranged fibrils, lining the lumen of the tubules, confirms that these are real structures. It is impossible to imagine how ice crystal formation could be responsible for arranging the structural elements into such regular features.

More recently, we have also studied the development of this structure from the neonatal to adult stages. At the early stages of development, when water content is higher than in the adult, no such tubular structure can be seen although similar preparation techniques had been applied. If these were artifactual structures then we would expect to see more of them in young tissue - not a complete absence. Indeed, their appearance seems to occur gradually, coinciding with the appearance of orthogonal fibrils and cells arranged in chondron columns.

We are only proposing that these distinctive tubular structures are present in rabbit tissue. We have also seen very similar structures in sheep tissue. Preliminary studies suggest that, in human and mouse tissue, the tubular arrangement is not as strongly developed - although many of the essential elements, such as the orthogonal fibrils, are present. There appear to be significant inter-specific variations, as well as significant developmental sequences of structural development. We have found that the age of the animal is a significant factor in determining whether tubular structures are detected. How these relate to phylogeny and/or life-style remains to be resolved. 